\title{
Squamous differentiation in pT1 bladder urothelial carcinoma predicts poor response for intravesical chemotherapy
}

\author{
Gang $\mathrm{Li}^{1, *}$, Jieping $\mathrm{Hu}^{2, *}$ and Yuanjie Niu${ }^{1}$ \\ ${ }^{1}$ Department of Urology, The Second Hospital of Tianjin Medical University, Tianjin Institute of Urology, Tianjin, 300211, \\ China \\ ${ }^{2}$ Department of Urology, The First Affiliated Hospital of Nanchang University, Nanchang, Jiangxi, 330006, China \\ *These authors contributed equally to this work
}

Correspondence to: Yuanjie Niu, email: niuyuanjie2012@sina.com

Keywords: squamous differentiation, pT1, bladder cancer, urothelial carcinoma, intravesical chemotherapy

Received: October 31, $2016 \quad$ Accepted: June 02, $2017 \quad$ Published: June 19, 2017

Copyright: Li et al. This is an open-access article distributed under the terms of the Creative Commons Attribution License 3.0 (CC BY 3.0), which permits unrestricted use, distribution, and reproduction in any medium, provided the original author and source are credited.

\section{ABSTRACT}

The role of squamous differentiation in pT1 bladder tumors in the response to intravesical chemotherapy was unknown. We performed a retrospective analysis of 213 pT1 bladder urothelial carcinoma patients with squamous differentiation (group1), the remaining 213 pT1 pure urothelial carcinoma served as controls (group2). All cases were treated with transurethral resection of bladder tumor and subsequent intravesical chemotherapy. Within a five-year period, the tumor recurrence rate was $75.1 \%(160 / 213)$ in group 1 and $64.3 \%(137 / 213)$ in group 2 . Tumor grade ( $H R=2.926, P=0.014)$, number of tumors $(H R=2.130, P=0.038)$, tumor size $(H R=2.748, P=0.031)$, and squamous differentiation $(H R=3.726, P=0.019)$ were found to be important prognostic factors. Subgroup analysis for high grade tumors was performed, finding that group 1 had higher recurrence rate $(50.3 \%$ vs $36.3 \%$; for group 2). Progression was found in 32.2\% (30/160) of group1 and $15.1 \%(11 / 137)$ of group2 $(P=0.011)$. Our data suggests that squamous differentiation is a predictor of poor response for intravesical chemotherapy, and that early radical cystectomy should be performed for high grade tumors, especially when dealing with recurrent cases.

\section{INTRODUCTION}

Urothelial carcinoma of the bladder (UCB) demonstrates a wide range of clinical behavior and morphology, including a peculiar capacity for divergent histologic differentiation, in which glandular and squamous have, in particular, been widely reported in UCB $[1,2]$. Focal squamous differentiation is seen most frequently with high-grade urothelial carcinoma, with some reporting this in $20-50 \%$ of urothelial carcinoma cases [3]. Keratinization and/or intercellular bridges are classical morphological features which can be found in squamous differentiation (SD) [4]. Squamous patterns detected in the histopathological specimen represents a negative prognostic factor $[5,6]$. It reported that the presence of a squamous component in patients with UCB may be associated with an ominous outcome treated with radical cystectomy [7]. An intensive case-control analysis suggested, however, that the outcomes of UCB patients who underwent radical cystectomy with SD were similar to those of patients facing pure UCB [1]. In a small study, patients with squamous tumors had lower five-year survival rates in comparison to patients with conventional high-grade tumors $(79.1 \%$ VS $89.5 \%$, respectively) treated with bacillus Calmette-Guerin (BCG) [8]. Moreover, patients with SD were more likely to have extravesical tumors and node positive disease $[9,10]$. These factors may alter the outcome of SD in patients. Thus, we conducted a retrospective study in order to observe tumor recurrence and progression to measure the efficacy of intravesical chemotherapy in $\mathrm{T} 1$ UCB with or without SD. 


\section{RESULTS}

\section{Patient characteristics}

The main characteristics of all patients are listed in Table 1. The mean age is $67.1 \pm 12.8$ years old for all patients, and $67.2 \pm 12.7$ and $67.0 \pm 12.8$ for group 2 and 1. respectively. Males composed $80.5 \%$ of all patients, and males accounted for $79.8 \%$ of group 2 and, $81.2 \%$ of group 1 . There was no significant difference in gender distribution $(P=0.716)$. In group 2, $128(60.1 \%)$ patients had high grade tumors, $61(28.6 \%)$ had multiple tumors, and $73(34.3 \%)$ had tumors larger than $3 \mathrm{~cm}$ in diameter. In group 1, these categories were $149(70.0 \%)$, 65 (30.5\%), 66 (31.0\%), respectively. Individually, tumor grading was statistically significant in comparison with group $2(P=0.042)$. The tumor recurrence rate over a five-year period was $64.3 \%$ (137 patients) in group 2 and $75.1 \%$ (160 patients) in group $1(P=0.020)$.

\section{Outcome for single-factor analysis}

Patients' follow-up period ranged from 4 to 112 months with an average of 62.2 months, and 297 (69.7\%) of all 426 patients had at least one recurrence, while 62 $(20.9 \%)$ of 297 patients progressed to muscle invasive disease during follow-up period. Progression was observed in $62(14.6 \%)$ patients, 20 of whom $(9.40 \%)$ were from group 2 and 42 of whom (19.7\%) were from group $1(P=0.004)$. A chi-square test was used to test the significant differences between single factors and recurrence. The results are shown in Table 2. Significant differences were found regarding tumor grade, size, SD, and the number of tumors in terms of recurrence.

\section{Multiple logistic regression analysis}

Factors that considered significant differences associated with recurrence according to the chi-square test were investigated using a multiple logistic regression analysis. Variables that represented tumor grade, number of tumors, and tumor size were included. Age, gender and SD were likewise not retained in the final models, as they were not significant at the 5\% level and their inclusion did not improve either model's discrimination or calibration. The following variables were identified to be of prognostic importance in the univariate analysis: grade $(\mathrm{HR}=2.926$, $P=0.014)$, number of tumors ( $\mathrm{HR}=2.130, P=0.038)$, tumor size $(\mathrm{HR}=2.748, P=0.031), \mathrm{SD}(\mathrm{HR}=3.726$, $P=0.019)$.

\section{Subgroup analysis variables with recurrence}

To explore the role of $\mathrm{SD}$, we compared the recurrence rate between group 2 and group 1 . As indicated in Table 3, 117 (78.5\%) of 149 high tumor grade patients had recurrence in group 1, while only $86(67.2 \%)$ of 128 high tumor grade patients had recurrence in group 2 $(P=0.041)$. Comparing group 2 with group 1 under the consideration of variables such as age and gender revealed no significant difference.

\section{Progression}

Sixty-two of 297 recurrent patients (20.9\%) progressed to muscle invasive disease. Forty-two (67.7\%) of 62 were SD patients, and 20 (32.3\%) patients were pure UCB, aAs indicated in Table 4. In the high-grade tumor group, 30.8\% (36 out of 117) patients with SD experienced tumor progression, whereas, only $17.4 \%$ (15 out of 86 ) group 2 patients had tumor progression $(P=0.034)$. In the low-grade tumor group, no significant difference was found between group 2 and group $1(P=0.749)$.

\section{DISCUSSION}

Worldwide, there are approximately 430,000 new bladder cancer diagnoses annually [11]. Urothelial carcinoma (UC) accounts for the majority of bladder cancer diagnoses and has a substantial impact on public health. The morphological diversity of $\mathrm{UC}$ is common. $\mathrm{UC}$ with $\mathrm{SD}$ is the most common variant which exists mainly in muscleinvasive UC, and there is mounting evidence revealed that SD may represent a precursor to invasive bladder cancer [8]. We detected SD in tumors via haematoxylineosin (H\&E) stains and immunohistochemical (IHC) and explored SD's association with tumor progression.

Our data indicated that the important prognostic factors for recurrence are: tumor grade, number of tumors, tumor size, and SD. SD was related to tumor recurrence and was also associated with tumor progression (Table 1), especially for high grade tumors (Table 3 ). We noted that patients in our study were all received intravesical chemotherapy with epirubicin or hydroxycamptothecine but not BCG, mitomycin C (MMC), or doxorubicin. In particular, BCG was not available in China because it has not been permitted by the China Food and Drug Administration. Our results were consistent with other studies which found that tumor grade, number of tumors, and tumor size affected disease outcome [12]. Rianne concluded that multiple tumors increased the risk of recurrences by $1.56-1.8$ in comparison with single tumors in Dutch cohort $(n=724)$ and Spanish cohort $(n=137)$ [13]. Similarly, in high-risk non-muscle-invasive bladder cancer patients treated with adjuvant BCG, tumor size was found to correlate with recurrence and progression [14]. To our best knowledge, it is the first time to prompt that SD can predict poor response to intravesical chemotherapy in pT1 bladder urothelial carcinoma.

It is well known that each histological variant has a different biology, data from 361 patients with invasive 
Table 1: The main characteristics of the patients

\begin{tabular}{|c|c|c|c|c|c|}
\hline \multicolumn{2}{|c|}{ Characteristic } & Total & PUCB & SUCB & $P$ value \\
\hline \multicolumn{2}{|c|}{ Cases, $n$} & 426 & 213 & 213 & \\
\hline \multicolumn{2}{|c|}{ Mean age (range), years } & $67.5(28-92)$ & $67.5(31-90)$ & $67.6(28-92)$ & 0.771 \\
\hline \multirow{2}{*}{ Gender, $n(\%)$} & M & 343 & 170 & 173 & \multirow{2}{*}{0.716} \\
\hline & $\mathrm{F}$ & 83 & 43 & 40 & \\
\hline \multirow{2}{*}{ Tumor grade, $n$} & Low & 149 & 85 & 64 & \multirow{2}{*}{0.042} \\
\hline & High & 277 & 128 & 149 & \\
\hline \multirow{2}{*}{ Tumor multiplicity, $n$} & No & 300 & 152 & 148 & \multirow{2}{*}{0.750} \\
\hline & Yes & 126 & 61 & 65 & \\
\hline \multirow{2}{*}{ Tumor size $(\mathrm{cm}), n$} & $<3$ & 287 & 140 & 147 & \multirow{2}{*}{0.535} \\
\hline & $\geq 3$ & 139 & 73 & 66 & \\
\hline \multirow{2}{*}{ Recurrence, $n$} & No & 129 & 76 & 53 & \multirow{2}{*}{0.020} \\
\hline & Yes & 297 & 137 & 160 & \\
\hline \multirow{2}{*}{ Progression, $n$} & No & 364 & 193 & 171 & \multirow{2}{*}{0.003} \\
\hline & Yes & 62 & 20 & 42 & \\
\hline
\end{tabular}

PUCB: pure urothelial carcinoma of bladder; SUCB: urothelial carcinoma of the bladder with SD.

Table 2: Chi square test of single factors

\begin{tabular}{|c|c|c|c|c|}
\hline Variable & Non Recurrence & Recurrence & $\lambda^{2}$ Value & $P$ Value \\
\hline \multicolumn{5}{|l|}{ Age } \\
\hline$\leq 67$ & 65 & 149 & \multirow{2}{*}{0.002} & \multirow{2}{*}{1.000} \\
\hline$>67$ & 64 & 148 & & \\
\hline \multicolumn{5}{|l|}{ Gender } \\
\hline Male & 104 & 239 & \multirow{2}{*}{0.001} & \multirow{2}{*}{1.000} \\
\hline Female & 25 & 58 & & \\
\hline \multicolumn{5}{|l|}{ Grade } \\
\hline Low & 55 & 94 & \multirow{2}{*}{4.773} & \multirow{2}{*}{0.035} \\
\hline High & 74 & 203 & & \\
\hline \multicolumn{5}{|l|}{ Number } \\
\hline Single & 102 & 198 & \multirow{2}{*}{6.642} & \multirow{2}{*}{0.011} \\
\hline Multiple & 27 & 99 & & \\
\hline \multicolumn{5}{|l|}{ Size } \\
\hline$<3 \mathrm{~cm}$ & 96 & 191 & \multirow{2}{*}{4.181} & \multirow{2}{*}{0.043} \\
\hline$\geq 3 \mathrm{~cm}$ & 33 & 106 & & \\
\hline \multicolumn{5}{|l|}{ Squamous } \\
\hline No & 76 & 137 & \multirow{2}{*}{5.882} & \multirow{2}{*}{0.020} \\
\hline Yes & 53 & 160 & & \\
\hline
\end{tabular}

bladder carcinoma suggests that conventional urothelial carcinoma, SD carcinoma and pure squamous carcinoma presented distinct outcomes, as mean survival periods were 73, 36, and 9.4 months, respectively [5]. We demonstrated that SD also predicts a poor response to intravesical chemotherapy with epirubicin or hydroxycamptothecine outcome in pT1 bladder cancer. When comparing patients with squamous cell carcinoma and those with SD who were treated with radical cystectomy and pelvic lymph node dissection, there appears to be no evidence of a 
Table 3: Chi square test in subgroup

\begin{tabular}{|c|c|c|c|c|c|}
\hline & \multicolumn{2}{|c|}{ PUCB } & \multicolumn{2}{|c|}{ SUCB } & \multirow[b]{2}{*}{$P$ value } \\
\hline & NR & $\mathbf{R}$ & NR & $\mathbf{R}$ & \\
\hline \multicolumn{6}{|l|}{ Age } \\
\hline$\leq 67$ & 37 & 68 & 28 & 81 & 0.139 \\
\hline$>67$ & 39 & 69 & 25 & 79 & 0.072 \\
\hline \multicolumn{6}{|l|}{ Gender } \\
\hline Male & 61 & 109 & 45 & 128 & 0.061 \\
\hline Female & 15 & 28 & 8 & 32 & 0.148 \\
\hline \multicolumn{6}{|l|}{ Grade } \\
\hline Low & 34 & 51 & 21 & 43 & 0.396 \\
\hline High & 42 & 86 & 32 & 117 & 0.041 \\
\hline \multicolumn{6}{|l|}{ Number } \\
\hline Single & 51 & 101 & 51 & 97 & 0.903 \\
\hline Multiple & 18 & 43 & 19 & 46 & 1.000 \\
\hline \multicolumn{6}{|l|}{ Size } \\
\hline$<3$ & 48 & 92 & 48 & 99 & 0.803 \\
\hline$\geq 3$ & 19 & 54 & 14 & 52 & 0.553 \\
\hline
\end{tabular}

NR: Non recurrence; R: recurrence; PUCB: pure urothelial carcinoma of bladder; SUCB: urothelial carcinoma of the bladder with SD.

Table 4: The progression patients in subgroup

\begin{tabular}{cccccc}
\hline & Low & & & High & Total \\
\hline & NP & P & NP & P & \\
PUCB & 46 & 5 & 37 & 6 & 94 \\
SUCB & 71 & 15 & 81 & 36 & 203 \\
Total & 117 & 20 & 118 & 42 & 297 \\
\hline
\end{tabular}

NP: Non progression; P: progression.

A

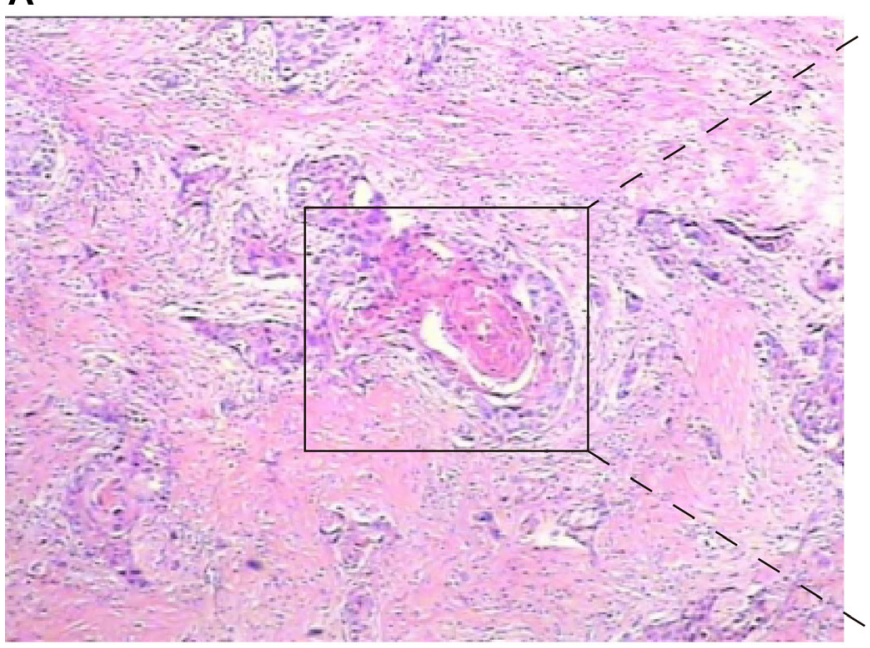

B

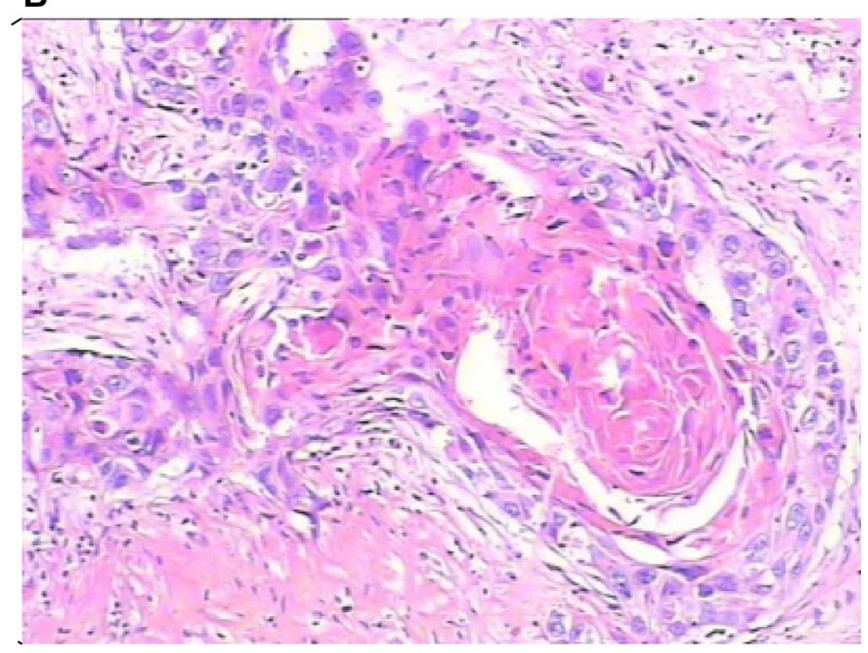

Figure 1: Hematoxylin and eosin staining of bladder urothelial carcinoma with SD ((A) Low magnification, (B) High magnification). 
difference in cancer specific survival or overall survival (log rank overall survival $P=0.6$, cancer specific survival $P=0.17$ ) [15]. SD in UCB were more likely to represent the biological characteristic of squamous cell carcinoma.

The strategy to battle UCB includes transurethral resection of bladder tumor (TURBT), intravesical chemotherapy, and radical cystectomy. Akdas reported a case of squamous cell carcinoma of the bladder induced by radiation therapy [16], thus radiation may avoid in pT1 UCB. For patients with pure squamous cell, adenocarcinoma, or small cell carcinoma, there is clear evidence to alter treatment paradigms [17]. In the subgroup of tumor grade, our data indicated that patients who suffered from high grade tumor accompanied by $\mathrm{SD}$ had a higher recurrence rate $(78.5 \%$ vs. $67.2 \%$, $P=0.041$, group 1 vs. group 2; Table 3) and progression rate $(30.8 \%$ vs. $17.4 \%, P=0.034$, group 1 vs. group 2 ; Table 4), supporting the idea that patients with high-risk non-muscle-invasive tumors and variant histology should be offered early cystectomy [18], especially for patients harboring SD.

Limitations of this study are noted as follows: (1) nonrandomized, retrospective analysis of a single center, which decreased the level of evidence; (2) the quality of TURBT operated by distinct surgery can affect the outcome; (3) two drugs (epirubicin and hydroxycamptothecine) applied in this study, distinct effect may achieved by different drug, we did not conducted detailed analysis; (4) there were some other intravesical chemotherapies like BCG or MMC in nonmuscle-invasive bladder cancer [19], but were not included in our study, Scosyrev concluded presence of squamous or glandular differentiation in locally advanced UC of the bladder does not confer resistance to methotrexate, vinblastine, doxorubicin and cisplatin (MVAC) [20], we do not ensure group 1 obtain same outcome in other chemotherapy. (5) we excluded the patients presence of CIS, which was an important prognostic factors [21].

\section{MATERIALS AND METHODS}

\section{Patients}

We retrospectively analyzed the clinical and pathological data of 213 patients who were diagnosed as pT1 bladder urothelial carcinoma with SD from May 2001 to Mar 2016 in our institution. Moreover, 213 pT1 urothelial carcinoma cases without SD were collected as control. The pathologic stage was based on the 2009 Union for International Cancer Control (UICC) TNM staging system. Tumor grade was based on the 2004 World Health Organization (WHO) grading system for non-invasive urothelial neoplasia [22]. Eligible cases meet the following criteria: (1) all patients should be confirmed as group 2 or UCB with SD by at least two pathologists; (2) all patients treated with TURBT; (3) patients who underwent schedule intravesical chemotherapy with epirubicin $50 \mathrm{mg}$ or hydroxycamptothecine $30 \mathrm{mg}$ once a week for 8 weeks and then once a month up to one year; (4) cystoscopy was given during the postoperative follow-up according to the European and US guidelines; (5) recurrence patients who received TURBT and intravesical chemotherapy in the low grade group and radical cystectomy in the high grade group. The exclusion criteria for our analysis were: (1) Ta, Tis or muscle invasive tumors; (2) imaging data indicated pelvic lymph nodes or distant organs metastases; (3) patients with a history of previous urothelial carcinoma or concomitant upper urinary tract urothelial carcinoma; (4) glandular, lymphoepithelioma-like, sarcomatoid or other differentiation; (5) Patients underwent partial or radical cystectomy; (6) patients underwent irregular intravesical chemotherapy or loss of follow up.

\section{Pathology}

All surgical specimens were submitted for pathological evaluation. Independent pathologic rereview of three representative slides from each patient was performed by two pathologists on all specimens in order to confirm reported pathologic findings. All specimens were assessed using H\&E staining (Figure 1), in which the presence of intercellular bridges or keratinization was indicative of SD. Uncertain pathological diagnosis was confirmed by IHC stains markers against CK20 and CK14.

\section{Statistical methods}

The chi-squared test and Student's $t$-test were used to evaluate the association between categorical and variables, respectively. Univariate and multivariate logistic regression models were used to analyze. All reported $P$ values were two-sided, and a $P$ value of $\leq 0.05$ was considered to indicate statistical significance. Statistical analysis was performed using SPSS software (Version 18).

\section{CONCLUSIONS}

$\mathrm{SD}$ in pT1 bladder urothelial carcinoma is a predictor of poor response for intravesical chemotherapy with epirubicin or hydroxycamptothecine. pT1 group 1 patients should receive early radical cystectomy to prevent disease progression especially for high tumor grade patients or recurrent cases.

\section{Author contributions}

G.L. designed the study, performed the data collection, contributed the figures and revised the manuscript. J.P.H wrote the manuscript, analyzed the data and discussed the results. Y.J.N. designed the study. All authors approved the final manuscript as submitted and agree to be accountable for all aspects of the work. 


\section{CONFLICTS OF INTEREST} interests.

The authors declare that they have no competing

\section{FUNDING}

This work was supported by Tianjin Municipal Natural Science Foundation (Grant 17JCYBJC26000) and Science and technology innovation fund projects of Tianjin Institute of Urology (number MNYB201503).

\section{REFERENCES}

1. Mitra AP, Bartsch CC, Bartsch G, Miranda G, Skinner EC, Daneshmand S. Does presence of squamous and glandular differentiation in urothelial carcinoma of the bladder at cystectomy portend poor prognosis? An intensive casecontrol analysis. Urol Oncol. 2014; 32:117-27. doi: 10.1016/j.urolonc.2012.08.017

2. Jankovic Velickovic L, Dolicanin Z, Hattori T, Pesic I, Djordjevic B, Stojanovic M, Stankovic J, Visnic M, Stefanovic V. Divergent SD in upper urothelial carcinomacomparative clinicopathological and molecular study. Pathol Oncol Res. 2011; 17:535-9. doi: 10.1007/s12253010-9343-x.

3. Shanks JH, Iczkowski KA. Divergent differentiation in urothelial carcinoma and other bladder cancer subtypes with selected mimics. Histopathology. 2009; 54:885-900. doi: 10.1111/j.1365-2559.2008.03167.x.

4. Gaisa NT, Braunschweig T, Reimer N, Bornemann J, Eltze E, Siegert S, Toma M, Villa L, Hartmann A, Knuechel R. Different immunohistochemical and ultrastructural phenotypes of SD in bladder cancer. Virchows Archiv. 2011; 458:301-12. doi: 10.1007/s00428010-1017-2.

5. Gluck G, Hortopan M, Stanculeanu D, Chirita M, Stoica R, Sinescu I. Comparative study of conventional urothelial carcinoma, SD carcinoma and pure squamous carcinoma in patients with invasive bladder tumors. J Med Life. 2014; 7:211-4.

6. Chalasani V, Chin JL, Izawa JI. Histologic variants of urothelial bladder cancer and nonurothelial histology in bladder cancer. Can Urol Assoc J. 2009 (Suppl 4); 3:S193-98.

7. Antunes AA, Nesrallah LJ, Dall'Oglio MF, Maluf CE, Camara C, Leite KR, Srougi M. The role of SD in patients with transitional cell carcinoma of the bladder treated with radical cystectomy. Int Braz J Urol. 2007; 33:339-45; discussion 46.

8. Gofrit ON, Yutkin V, Shapiro A, Pizov G, Zorn KC, Hidas G, Gielchinsky I, Duvdevani M, Landau EH, Pode D. The Response of Variant Histology Bladder Cancer to Intravesical Immunotherapy Compared to Conventional Cancer. Front Oncol. 2016; 6:43. doi: 10.3389/fonc.2016.00043.

9. Kim SP, Frank I, Cheville JC, Thompson RH, Weight CJ,
Thapa P, Boorjian SA. The impact of squamous and glandular differentiation on survival after radical cystectomy for urothelial carcinoma. J Urol. 2012; 188:405-9. doi: 10.1016/j.juro.2012.04.020.

10. Li G, Song H, Wang J, Bao Y, Niu Y. Poor prognostic value of lymphovascular invasion for pT1 urothelial carcinoma with SD in bladder cancer. Sci Rep. 2016; 6:27586. doi: $10.1038 /$ srep 27586 .

11. Antoni S, Ferlay J, Soerjomataram I, Znaor A, Jemal A, Bray F. Bladder Cancer Incidence and Mortality: A Global Overview and Recent Trends. Eur Urol. 2016. doi: 10.1016/j.eururo.2016.06.010.

12. Sylvester RJ, van der Meijden APM, Oosterlinck W, Witjes JA, Bouffioux C, Denis L, Newling DWW, Kurth K. Predicting recurrence and progression in individual patients with stage Ta T1 bladder cancer using EORTC risk tables: A combined analysis of 2596 patients from seven EORTC trials. European Urology. 2006; 49:466-77. doi: 10.1016/j. eururo.2005.12.031.

13. Lammers RJ, Hendriks JC, Rodriguez Faba OR, Witjes WP, Palou J, Witjes JA. Prediction model for recurrence probabilities after intravesical chemotherapy in patients with intermediate-risk non-muscle-invasive bladder cancer, including external validation. World J Urol. 2016; 34:173-80. doi: 10.1007/s00345-015-1598-0.

14. Zachos I, Tzortzis V, Mitrakas L, Samarinas M, Karatzas A, Gravas S, Vandoros GP, Melekos MD, Papavassiliou AG. Tumor size and T stage correlate independently with recurrence and progression in high-risk non-muscle-invasive bladder cancer patients treated with adjuvant BCG. Tumour Biol. 2014; 35:4185-9. doi: 10.1007/s13277-013-1547-8.

15. Ehdaie B, Maschino A, Shariat SF, Rioja J, Hamilton RJ, Lowrance WT, Poon SA, Al-Ahmadie HA, Herr HW. Comparative outcomes of pure squamous cell carcinoma and urothelial carcinoma with SD in patients treated with radical cystectomy. J Urol. 2012; 187:74-9. doi: 10.1016/j. juro.2011.09.056.

16. Akdas A, Turkeri L. The impact of squamous metaplasia in transitional cell carcinoma of the bladder. Int Urol Nephrol. $1991 ; 23: 333-6$.

17. Willis DL, Porten SP, Kamat AM. Should histologic variants alter definitive treatment of bladder cancer? Current Opinion in Urology. 2013; 23:435-43. doi: 10.1097/ MOU.0b013e328363e415.

18. Porten SP, Willis D, Kamat AM. Variant histology: role in management and prognosis of nonmuscle invasive bladder cancer. Curr Opin Urol. 2014; 24:517-23. doi: 10.1097/ MOU.0000000000000089.

19. Porten SP, Leapman MS, Greene KL. Intravesical chemotherapy in non-muscle-invasive bladder cancer. Indian J Urol. 2015; 31:297-303. doi: 10.4103/0970-1591.166446.

20. Scosyrev E, Ely BW, Messing EM, Speights VO, Grossman HB, Wood DP, White RWD, Vogelzang NJ, Trump DL, Natale RB, Tangen CM, Crawford ED, Thompson IM. Do mixed histological features affect survival 
benefit from neoadjuvant platinum-based combination chemotherapy in patients with locally advanced bladder cancer? A secondary analysis of Southwest Oncology Group-Directed Intergroup Study (S8710). BJU Int. 2011; 108:693-9. doi: 10.1111/j.1464-410X.2010.09900.x.

21. Sylvester RJ. Natural history, recurrence, and progression in superficial bladder cancer. ScientificWorldJournal. 2006; 6:2617-25. doi: 10.1100/tsw.2006.404.
22. Humphrey PA, Moch H, Cubilla AL, Ulbright TM, Reuter VE. The 2016 WHO Classification of Tumours of the Urinary System and Male Genital Organs-Part B: Prostate and Bladder Tumours. Eur Urol. 2016. doi: 10.1016/j. eururo.2016.02.028. 